\title{
A retrospective audit of hyperlipidaemia management following acute coronary syndromes
}

\author{
Authors: Kaung Lwin, ${ }^{\mathrm{A}}$ Hnin Hay Mar, ${ }^{\mathrm{B}}$ Zaw Aung ${ }^{\mathrm{A}}$ and Chee Khoo ${ }^{\mathrm{A}}$
}

\section{Introduction}

Control of hypercholesterolaemia is a highly effective way of reducing cardiovascular events in patients at high risk. Repeat cholesterol measurement is good practice in lipid management to optimise treatment but is poorly performed. Following National Institute for Health and Care Excellence (NICE) approval of proprotein convertase subtisilin/kexin 9 inhibitor (PCSK9i), there is a need to identify patients who are eligible for this treatment.

\section{Objectives}

To determine performance in lipid measurement 3 months following initiation of lipid therapy. ${ }^{1}$ To determine whether all patients with an indication for a PCSK9i following acute coronary syndrome (ACS) were being identified. ${ }^{2}$

\section{Methods}

A retrospective study evaluated ACS patients using Myocardial Infarction National Audit Project (MINAP) registries from Royal Stoke Hospital (January 2014 - December 2016). MINAP included patients who were admitted directly to Royal Stoke Hospital and transferred from nearby district general hospitals; Leighton, Macclesfield, Shrewsbury and Telford, Burton area following ACS for treatment. All patients whose initial serum cholesterol $>7.3$ on admission were identified as sample population from MINAP data. Total cholesterol and LDL levels were recorded during admission and post-discharge.

\section{Results}

There was a total 3,548 patients in MINAP (January 2014 December 2016). 75 patients (2.1\%) were identified as sample population. Eight patients did not have any record of lipid profiles on our system and GPs did not wish to engage in supplying information. Six patients deceased. 61 patients were analysed. Mean age was 57 (Range 27-80). Mean cholesterol was 7.85. LDL-C was poorly measured as there were only $5 \%(n=2)$ measured on admission and $40 \%(n=24)$ at 3 month after discharge. Serum cholesterol were repeated in $58 \%(n=35)$ of the patients at

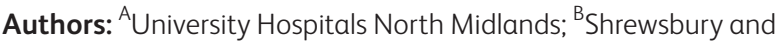
Telford NHS Trust
3 month following event. We identified two high risk patients (total one event) met for PCSK9 inhibitor as LDL-C was persistently $>4$, and two high risk patients whose last LDL-C $>4$ despite maximum statin and one very high risk patients (two or more event) whose last LDL-C >3.5, potential meet for PCSK9i. There were two high risk patients already started on PCSK9i and their LDL-C were reduced significantly.

\section{Conclusions}

Lipid profiles, especially LDL-C, were poorly done on admission and following discharge. This could be improved by repeating lipid profiles when patients attend for cardiac rehabilitation and recommending GPs to repeat the lipid profiles following discharge. A small number of patients were identified as being eligible for PCSK9i. Failure to repeat LDL levels in patients following discharge from hospital is likely to explain this. There was also possible underreporting of comorbid vascular diseases within the MINAP database may prevent the identification of patients at very high risk. We re-established the routine practice of measuring lipid profile in our cardiology wards on admission, repeating during cardiac rehabilitation, and recommending GPs to measure following discharge.

\section{References}

1 National Institute for Health and Care Excellence. Evolocumab for treating primary hypercholesterolaemia and mixed dyslipidaemia. Technology appraisal guidance [TA394]. London: NICE, 2019. www.nice.org.uk/guidance/ta394 [Accessed 25 February 2019].

2 National Institute for Health and Care Excellence. Cardiovascular disease: risk assessment and reduction, including lipid modification. Clinical guideline [CG181]. London: NICE. 2019. www.nice.org.uk/ guidance/cg181 [Accessed 25 February 2019]. 